\title{
High Density Nanostructured Soft Ferrites Prepared by High Pressure Field Assisted Sintering Technique
}

\author{
M. Petrecca ${ }^{1}$, M. Albino ${ }^{1}$, I. G. Tredici ${ }^{3}$, U. Anselmi-Tamburini ${ }^{3}$, \\ M. Passaponti ${ }^{1}$, A. Caneschi ${ }^{1}$, and C. Sangregorio ${ }^{1,2, *}$ \\ ${ }^{1}$ Dip. di Chimica, "Ugo Schiff”, Università degli Studi di Firenze, via della Lastruccia 3-13, \\ I-50019 Sesto Fiorentino, and Consorzio INSTM, Italy \\ ${ }^{2}$ CNR-ICCOM, via Madonna del Piano 10, I-50019 Sesto Fiorentino, and Consorzio INSTM, Italy \\ ${ }^{3}$ Dip. di Chimica, Università degli Studi di Pavia, v.le Taramelli 12, I-27100 Pavia, Italy
}

\begin{abstract}
The synthesis of highly compacted, nanostructured soft magnets is highly desirable due to their promising properties for the development of electronic devices working at frequency higher than $2 \mathrm{MHz}$. In this work we investigated the potentiality of High Pressure Field Assisted Sintering Technique (HP-FAST). To this aim, we first synthesized soft $\mathrm{Mn}-\mathrm{Zn}$ ferrite magnetic nanoparticles (MNPs) through an easy-scalable, eco-friendly strategy based on aqueous co-precipitation in basic media, starting from transition metal chlorides. Powder X-ray diffraction (PXRD) and Transmission Electron Microscopy (TEM) analyses evidenced the formation of crystalline nanoparticles with the cubic spinel structure and average crystal size of $7.5 \mathrm{~nm}$. Standard magnetometric measurements showed a saturation magnetization value of ca. $56 \mathrm{emu} / \mathrm{g}$ and no magnetic irreversibility at room temperature. The MNPs were then compacted applying an uniaxial pressure over a toroidal shaped die. In order to obtain a material with a density close to the bulk one, the as-prepared green toroids underwent either a classic sintering treatment, obtaining a microstructured system, or to High Pressure Field Assisted Sintering Technique (HP-FAST), which allowed for preserving the nanostructure. The relative permeability and core losses of the toroidal samples were evaluated in the frequency range 1-2 $\mathrm{MHz}$ using an in-house built setup. The comparison of the behavior of samples obtained by the two different sintering approaches showed the nanostructured samples had a much smaller relative magnetic permeability (ten times lower than the microstructured sample) and, consequently, higher core losses. However, when samples with similar $\mu_{r}$ were compared, a significant decrease of core losses at the larger frequencies was observed. This result suggests HP-FAST is a very promising approach to prepare high density nanostructured soft magnetic materials.
\end{abstract}

Keywords: Mn-Zn Ferrite, Soft Magnetic Materials, Nanostructure, Core Loss.

\section{INTRODUCTION}

Soft magnetic materials represent an economically relevant key element in modern technology, where they are widely used to realize electronic devices such as transformers, choke coils, inductors and high-frequency power electronics. $^{1,2}$ All these devices, indeed, comprise a soft magnetic core inserted in an electric circuit, working both in direct current (DC) or alternating current (AC). ${ }^{3}$ The constant demand for increasing the efficiency and miniaturizing the size of electronic devices requires the use of

*Author to whom correspondence should be addressed. ever increasing frequencies, which, at the same time makes energy dissipation more and more critical. The reduction of the energy dissipation as heat (power losses) is indeed, at present, the keystone for the effective use of soft magnetic cores in electronic devices. In the range of frequency of few $\mathrm{MHz}$ the main contributions to power losses are hysteresis, conductivity (eddy current loss) and residual losses. ${ }^{4}$ Typical approaches to reduces hysteresis losses are based on increasing the magnetic permeability and/or decreasing the anisotropy by fine tuning the composition of ferrites and alloys, increasing the grain size of bulk metal alloys or using amorphous alloys. ${ }^{5-7}$ On the other hand the reduction of eddy currents requires the 
enhancement of the electrical resisitivity, which can be obtained by lamination or reducing thickness. ${ }^{6,7}$

Within this respect, the reduction of the grain size to the nanoscale can be an effective strategy to decrease power losses, as it can allow for the reduction of both hysteresis losses and eddy currents at high frequency. ${ }^{8-10}$ Although from this point of view magnetic nanoparticles are very promising, their utilization poses the additional challenge of transforming a nanopowders into a highly dense, compact material, which can be shaped in any desired form, without a substantial loss of their unique magnetic properties.

Thanks to the high initial magnetic permeability and low core losses, Mn-Zn ferrites are particularly suited for the production of soft magnetic cores. ${ }^{4}$ Ferrites are usually produced by ceramic process, which involves the use of high temperature and long sintering time, usually carried out in conventional furnaces. However, if the final target is a nanostructured material both the synthesis temperature and the sintering parameters must be careful tuned to avoid uncontrolled grain growth. In the recent past many synthetic methods have been proposed to synthesize nanocrystalline ferrite, such as thermal decomposition, ${ }^{11}$ micro-emulsion, ${ }^{12}$ hydrothermal precipitation ${ }^{13}$ and coprecipitation, the latter being particularly appealing due to its simplicity and easiness of scale-up, although at expenses of the quality of the nanocrystals. Similarly, a lot of efforts have been devoted in developing suitable sintering technique able to preserve the nanostructuration: hot pressing sintering, hot isostatic pressing sintering and microwave sintering are some of the most known examples. Recently, attention has focused on some innovative sintering techniques, such as spark plasma sintering (SPS), plasma activated sintering (PAS) and field assisted sintering (FAST). In particular, SPS/FAST has attracted large attention since it allows for rapid heating, short holding time, high sintering density, small grain size and easy manipulation. ${ }^{14}$

In this work, we discuss the potentiality of HP-FAST to obtain nanostructured, high density compacted toroids of $\mathrm{Mn}-\mathrm{Zn}$ ferrites with low core losses. $\mathrm{Mn}-\mathrm{Zn}$ ferrite nanopowders were prepared through a simple method based on co-precipitation, which can be easily scaled-up to industrial production at low cost. The as-synthesized nanomaterials were then compacted to obtain toroids with quasi-bulk density both by conventional heating and by HP-FAST treatment, with the purpose to compare the power losses of the materials.

\section{EXPERIMENTAL DETAILS}

Nanocrystalline $\mathrm{Mn}-\mathrm{Zn}$ ferrite powder of formula $\mathrm{Mn}_{0.6} \mathrm{Zn}_{0.4} \mathrm{Fe}_{2} \mathrm{O}_{4}$, was synthesized by the co-precipitation method. ${ }^{15}$ Reaction grade ferric chloride hydrate, $\mathrm{FeCl}_{3}$. $6 \mathrm{H}_{2} \mathrm{O}$, manganese chloride hydrate, $\mathrm{MnCl}_{2} \cdot 4 \mathrm{H}_{2} \mathrm{O}$, zinc chloride, $\mathrm{ZnCl}_{2}$, and $\mathrm{NH}_{4} \mathrm{OH} 33 \%$ w/w were purchased from Sigma Aldrich and used without further purification. The molar ratio of starting transition metal chlorides was adjusted according to the desired stoichiometry. Initially, the $\mathrm{pH}$ of a solution of $\mathrm{Fe}^{3+}$, obtained by dissolving 2 equivalent of $\mathrm{FeCl}_{3} \cdot 6 \mathrm{H}_{2} \mathrm{O}$ in demineralized water was adjusted to 8 using $\mathrm{NH}_{4} \mathrm{OH} 33 \%$ in weight, in order to obtain the complete precipitation of ferric hydroxide as a brown slurry. The precipitated was collected through filtration on a Buchner funnel, washed several times with demineralized water and then added to a solution of $\mathrm{MnCl}_{2}$. $4 \mathrm{H}_{2} \mathrm{O}$ and $\mathrm{ZnCl}_{2}$ in demineralized water whose $\mathrm{pH}$ was adjusted to 12 by using $\mathrm{NH}_{4} \mathrm{OH} 33 \%$. The resulting dark brown precipitate, composed by transition metal hydroxides was heated to reflux, under $\mathrm{N}_{2}$ flow and magnetic stirring, and kept at $100{ }^{\circ} \mathrm{C}$ for 2 hours. Then, the asobtained black suspension was cooled to room temperature and the black precipitate was collected using a permanent magnet. The magnetic nanoparticles were washed several times with water, ethanol and acetone and dried overnight at $80^{\circ} \mathrm{C}$.

The sintering procedure was carried out in an inhouse built HP-FAST apparatus, allowing heating rate of $100{ }^{\circ} \mathrm{C} / \mathrm{min}$ up to $1000{ }^{\circ} \mathrm{C}$ under vacuum. In order to obtain a final product with a toroid-like shape, green toroids were prepared from nanopowders using a graphite die with $10 \mathrm{~mm}$ outer and $4 \mathrm{~mm}$ inner diameters. In order to preserve the toroidal shape during the sintering, the inner hole was filled with a rounded graphite sheet. The toroids were then inserted into a graphite die of $15 \mathrm{~mm}$ outer diameter and exposed to the HP-FAST treatment. Several samples were prepared by varying the sintering temperature, $T_{\text {sin }}$, and pressure as key parameters.

For the purpose of comparison $\mathrm{Mn}-\mathrm{Zn}$ ferrite toroids were also prepared by classical sintering procedure, i.e., pressing the nanopowder with a die of $15 \mathrm{~mm}$ outer and $8 \mathrm{~mm}$ inner diameters and exposing it to heating treatments. These treatments were carried out in tubular furnace under inert atmosphere $\left(\mathrm{N}_{2}\right.$ flow) at different sintering temperature. During the process the heating rate $\left(10^{\circ} \mathrm{C} / \mathrm{min}\right.$ ) and dwell time (2 hours) were kept constant. The density of the samples was measured through the classic Archimedean method, using the following equation:

$$
d=\frac{d_{\mathrm{water}} m_{\mathrm{air}}}{m_{\mathrm{air}}-m_{\mathrm{water}}}
$$

were $d_{\text {water }}$ is the density of water at the measuring temperature and $m_{\text {air }}$ and $m_{\text {water }}$ are the sample weight in air and water, respectively, measured with a microbalance. The density values are reported as normalized to the bulk value $\left(5.07 \mathrm{~g} / \mathrm{cm}^{3}\right)$.

The crystallographic structure of both as-synthesized and sintered samples was analyzed by powder XRD using a Bruker Advance D8 diffractometer equipped with $\mathrm{Cu}$ $\mathrm{K} \alpha$ radiation and operating in $\theta-\theta$ Bragg-Brentano geometry at $40 \mathrm{kV}$ and $40 \mathrm{~mA}$. The size and morphology of 
nanoparticles was investigated by TEM using a CM12 Philips microscope. Magnetic measurements were carried out on tightly packed, randomly oriented powders using a vibrating sample magnetometer (VSM) Quantum Design PPMS. The relative magnetic permeability $\left(\mu_{r}\right)$ in AC field was evaluated by measuring the inductance $(L)$, using a "Ronde \& Schwarz ZNB8 Vector Network Analyser 9 kHz ...8 GHz", calibrated with a Rosenberg RPC $3.5 \mathrm{~mm}$ calibration kit, in the range $100 \mathrm{kHz}-10 \mathrm{MHz} . \mu_{r}$ values were obtained using the following equation

$$
\mu_{r}=\frac{2 \pi L}{\mu_{0} h N^{2} \ln \left(d_{\text {out }} / d_{\text {in }}\right)}
$$

were $d_{\text {out }}, d_{\text {in }}$ and $h$ are geometrical parameters of the toroid (outer diameter, inner diameter and height, respectively) $N$, is the number of wounds of the $0.2 \mathrm{~mm}$ copper wire used for inducing the field $B$ and $\mu_{0}$ is the vacuum permeability. The core losses were measured using an inhouse built setup comprising a multifrequency wave generator, which generates an electromagnetic square wave in the sample in the frequency range of 1-2 $\mathrm{MHz}$, with a tuneable duty cycle. The tune of duty cycle allows the temporization of the square wave as a function of frequency in order to achieve the zero volt switching regime in the primary and secondary circuit. Then, a tension $(V)$ is applied on the sample by a power supply and the current flowing in the coil, $I$, is measured by the power supply itself. To obtain a more precise result, the power losses due to the circuits and to the measuring system are measured by an oscilloscope and subtracted from the total losses. Operatively, considering the number of wire turns $(N)$, the dimension of the toroid, from which we can estimate the area crossed by the $B$ flux $\left(A_{e}\right.$, expressed in $\left.\mathrm{cm}^{2}\right)$, and the frequency used, from which the activation time of tension difference is calculated $\left(T_{\text {on }}=1 / 2 \omega\right.$, expressed in seconds), it is possible to adjust the voltage to produce a certain $B$ in the sample, using the following equation:

$$
V_{\text {in }}=\frac{2 B A_{e} N}{T_{\text {on }}}
$$
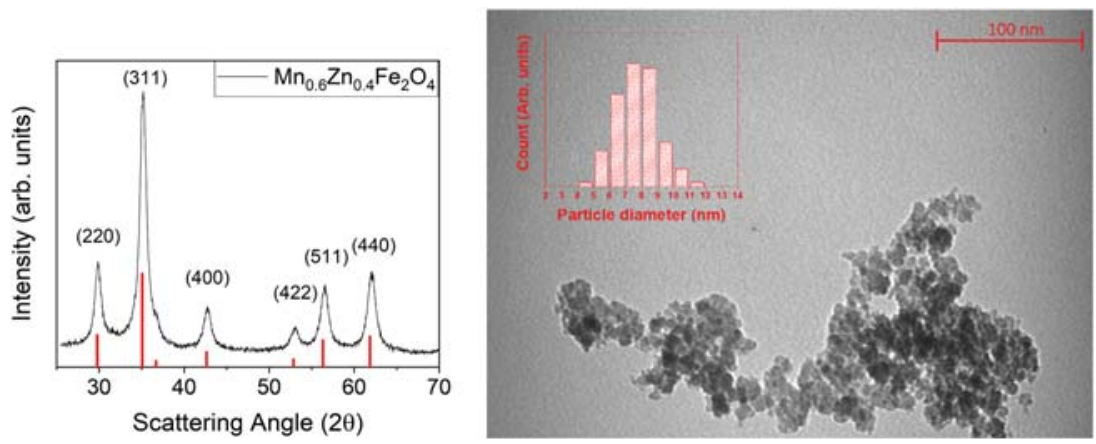

Figure 1. Left: Experimental XRD pattern of the Mn-Zn ferrite powders. Red bars represent the reference pattern for Mn-Zn ferrite (Ref. JCPDS PDF \#74-2401); Right: Representative TEM bright field image of the nanopowder. Scale bar $=100 \mathrm{~nm}$. In the inset the size distribution evaluated over more than 100 nanoparticles is shown. 
Table I. Sintering temperature and pressure, and properties of the corresponding material: Density relative to the bulk; lattice parameter and crystal size evaluated from XRD patterns.

\begin{tabular}{lccccc}
\hline Sample & $\begin{array}{c}\text { Temperature } \\
\text { (C) }\end{array}$ & $\begin{array}{c}\text { Sintering } \\
\text { pressure } \\
(\mathrm{MPa})\end{array}$ & $\begin{array}{c}\text { Relative } \\
\text { density } \\
(\%)\end{array}$ & $\begin{array}{c}\text { Lattice } \\
\text { parameter } \\
(\mathrm{nm})\end{array}$ & $\begin{array}{c}\text { Crystal } \\
\text { size } \\
(\mathrm{nm})\end{array}$ \\
\hline F500 & 500 & 77 & 69 & $0.8467(2)$ & 40 \\
F600 & 600 & 68 & 77 & $0.8464(2)$ & 62 \\
F700 & 700 & 71 & 99 & $0.8466(2)$ & 65 \\
F800 & 800 & 74 & 97 & $0.8468(2)$ & 100 \\
F900 & 900 & 92 & 94 & $0.8470(2)$ & $>300$ \\
F1000 & 1000 & 86 & 96 & $0.8469(2)$ & $>300$ \\
\hline
\end{tabular}

field cooling (FC) procedures in the presence of a 50 Oe applied field.

As observed from Figure 2, the ZFC curve of Mn-Zn ferrite nanoparticles presented a maximum at a temperature, $T_{\max }$, close to $200 \mathrm{~K}$. As a first approximation, $T_{\max }$ can be identified with the blocking temperature, $T_{B}$, of the system. The observed value is higher than that commonly reported in the literature for weakly interacting manganese zinc doped ferrite nanoparticles, ca $100 \mathrm{~K} .{ }^{18}$ The shift to higher temperature can be ascribed to the large interaction existing between nanoparticles, enhanced by the absence of any capping agent on the particle surface. The hysteresis loop was recorded at $300 \mathrm{~K}$, applying an external field between -50 and $+50 \mathrm{kOe}$ on the pressed powder pellet. The saturation magnetization, $M_{S}$, obtained by fitting the curve to a Langevin function, was $56 \mathrm{emu} / \mathrm{g}$, comparable with the $M_{S}$ value reported in the literature for nanoparticles with the same composition, obtained by a similar synthetic strategy. ${ }^{17}$ At room temperature no magnetic irreversibility was observed, at least within the resolution limit of the instrument (ca. 50 Oe). Therefore, as indeed suggested by ZFC-FC curves too, the synthesized nanoparticles are in the superparamagnetic regime at room temperature, a property which may determine the reduction of losses associated to the hysteretic behaviour.

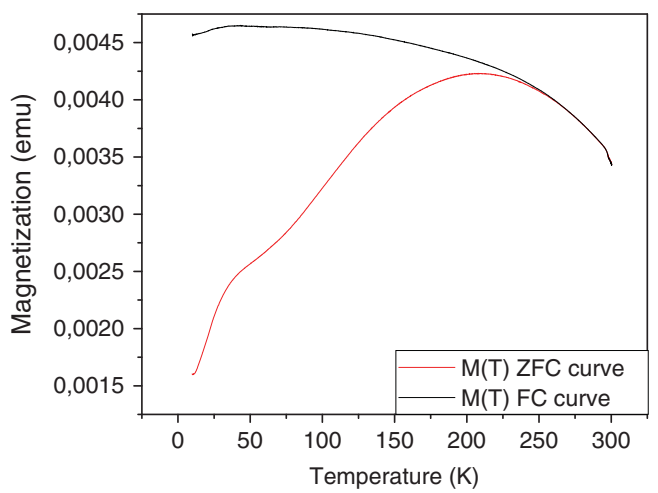

The $\mathrm{Mn}-\mathrm{Zn}$ ferrite nanopowders were then pressed and exposed to HP-FAST heating treatment at various temperature and pressure, with the aim of obtaining final material with a quasi-bulk density while preserving the grain nanostructure. In Table I are reported the different sintering conditions used, the density relative to the bulk, and crystallographic parameters (lattice and crystal size) of the corresponding materials. During the process the heating rate $\left(100{ }^{\circ} \mathrm{C} / \mathrm{min}\right)$ and dwell time at the sintering temperature (5 min.) were kept constant. In the following, sample are named referring to the temperature used during the sintering process.

XRD analysis revealed that the cubic spinel structure was preserved at any sintering temperature, while the lattice parameter was almost constant and equal to that of the starting nanoparticles, with a value of $0.8467+/$ $-0.0003 \mathrm{~nm}$, suggesting the composition remained unaltered during the thermal treatment. The preservation of the initial composition of the ferrite was confirmed by X-ray Fluorescence analysis. The grain size increased with $T_{\text {sin }}$ and reached few hundreds of nm for $T_{\text {sin }} \geq 900{ }^{\circ} \mathrm{C}$. For $T_{\text {sin }} \geq 700{ }^{\circ} \mathrm{C}$ the samples exhibited density value close to the bulk one, the largest values being observed for $T_{\text {sin }}=$ $700{ }^{\circ} \mathrm{C}(99 \%)$ and $T_{\sin }=800{ }^{\circ} \mathrm{C}(97 \%)$. Considering the aim of this work, we therefore focused our attention on samples F700 and F800, which displayed the highest density and crystal sizes which are still in the single domain regime. ${ }^{19,20}$ These two conditions should indeed lead to lower power losses thanks to increased magnetic permeability (density) and decreased eddy currents (grain size), despite of a possible increase of hysteresis losses, which, for a soft material such this are expected to be low. The magnetic permeability and core loss of these two samples were investigated in the frequency range $1-2 \mathrm{MHz}$ inducing a magnetic flux B of $500 \mathrm{G}$. The obtained results are shown in Figure 3 and are compared to the behavior of a sample obtained through a classic approach, i.e., sintered at $1200{ }^{\circ} \mathrm{C}$, following the procedure described in the experimental part. This sample presented a density of $98 \%$

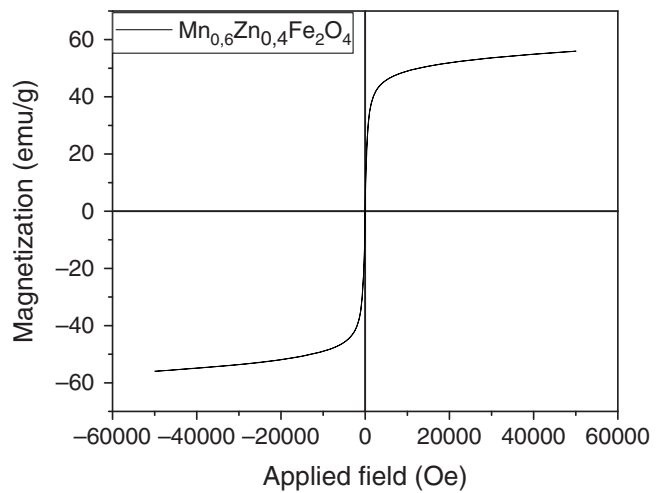

Figure 2. Left: Temperature dependence of the zero field cooled (red) and field cooled (black) magnetizations; Right: Room temperature hysteresis loop of the as-synthesized powder. 

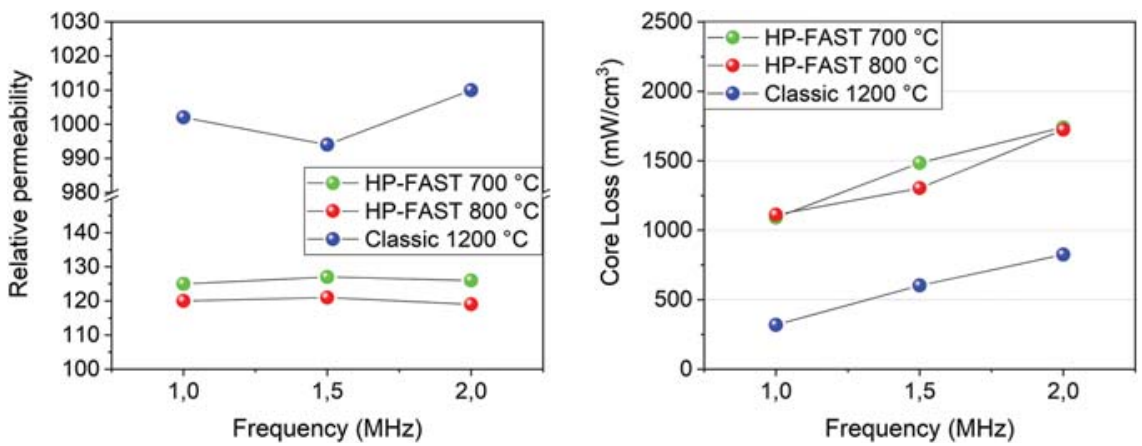

Figure 3. Relative permeability (left) and core loss (right) as a function of frequency for samples F700 (green) and F800 (red). For comparison the properties of a sample obtained by classic sintering approach at $1200{ }^{\circ} \mathrm{C}$ are also reported (blue).

with respect to the bulk and grain size in the micrometric scale.

From the data it clearly emerges that samples prepared by HP-FAST had lower relative permeability (ca. 120) and larger core loss, ca. $1500 \mathrm{~mW} / \mathrm{cm}^{3}$ at $2 \mathrm{MHz}$, with respect to the one prepared by classical sintering $\left(\mu_{r} 1000\right.$ and core loss ca. $\left.950 \mathrm{~mW} / \mathrm{cm}^{3}\right)$. This result shows that the microstructure obtained by the classic sintering procedure lead to a quasi-bulk dense product, and thus determined improved performances with respect to the nanostructured samples, both in terms of relative magnetic permeability and core losses.

However, it should be considered that core loss and relative permeability are strongly correlated. In fact, the magnetic flux, $B$, induced in the toroid is proportional to the magnetic permeability, which depends on the intrinsic magnetic properties of the material and on the quality of the toroid (cracking or inhomogeneities decrease the magnetic permeability expelling the magnetic flux). The correct comparison of power losses of different materials requires that the same $\mathrm{B}$ is induced during the measurement. However, if two samples with very different relative permeability are compared, the matching of this condition requires increasing either the applied voltage or, alternatively the number of wounds in the sample with lower $\mu_{r}$, which, in turn, produces an increase of eddy currents, thus hampering the proper evaluation of the power losses. Since the difference in magnetic permeability may simply arise from a difference in the toroid quality, this means that the latter may produce a variation in the final core losses, independently of the intrinsic efficiency of a given material. This problem can be avoided if samples with almost the same relative permeability are considered. Therefore, in Figure 4 the core losses of F700 and F800 $\left(\mu_{r}=120\right)$ were compared to that of a sample prepared through classical technique, sintered at $1000{ }^{\circ} \mathrm{C}$, following the procedure described in the experimental part, and which presented a similar relative permeability $\left(\mu_{r}=115\right)$, relative density of $93 \%$ and microstructured grains.

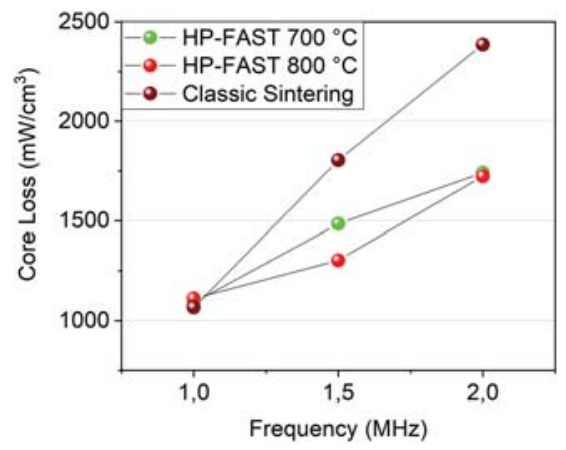

Figure 4. Core losses of samples F700 (green) and F800 (red) compared to classically sintered sample $\left(T=1000{ }^{\circ} \mathrm{C}\right)$ with similar relative permeability (brown).

A clear difference between the samples emerged on increasing frequency: indeed, while at $1 \mathrm{MHz}$ the core losses were almost the same for all the samples, at 1.5 $\mathrm{MHz}$ and $2 \mathrm{MHz}$ the classically sintered sample showed a higher core loss $(121 \%$ and $138 \%$ at $1.5 \mathrm{MHz}$ and $137 \%$ and $138 \%$ at $2 \mathrm{MHz}$ of $\mathrm{F} 700$ and $\mathrm{F} 800$, respectively). This result is consistent with the theoretical behaviour described in the literature, ${ }^{21}$ which predicts that the eddy current contribution to the energy loss per cycle increases with frequency, while the hysteresis one is frequency independent. We can therefore conclude that the nanostructuration of the grains allows for reducing core losses arising from eddy currents. In fact, eddy current losses scale with the square power of particle diameter ${ }^{22}$ and become almost negligible when the size of the grains approaches the nanoscale.

\section{CONCLUSION}

$\mathrm{Mn}-\mathrm{Zn}$ ferrite nanoparticles were prepared through a simple, easily scalable and eco-friendly method based on aqueous co-precipitation, starting from transition metal chlorides and using ammonium hydroxide as basic media. The nanometric material was characterized for the crystallographic, morphological and magnetic properties and 
was then used to prepare quasi-bulk toroids, with the aim of obtaining a high density material while preserving the nanometric structure. The morpho-structural characterization of the sintered samples showed that HP-FAST with sintering temperature in the $700-800{ }^{\circ} \mathrm{C}$ range, is a suitable technique to prepare high density toroids of soft ferrite grains with size in the nanometric range. Thanks to the nanostructure these samples exhibited at high frequency (1.5 and $2 \mathrm{MHz}$ ) smaller power losses when compared to a sample obtained by classical sintering and with similar magnetic permeability. The result can be ascribed to the reduction of eddy current contribution occurring when the size of the grains is reduced to the nanometric scale.

To conclude, High Pressure Field Assisted Sintering Technique proved to be very promising for the preparation of soft magnetic, highly compacted nanostructured materials. Moreover, it should be considered that in our case, the requirement of producing a toroidal shape suitable for measuring core loss with our apparatus, introduced several defects in the bulk structure as fractures and inhomogeneities, which affected the relative magnetic permeability, and, as discussed above, increased core losses, independently of the intrinsic properties of the material. However, these requirement does not hold for the standard application of the material in electronic devices where simpler shape (plate, cylinder, disks, etc...) are commonly used. It can be argued that realizing such simpler shape would lead to much better performances of the final system, reducing the number of fractures and inhomogeneities. A further improvement can be obtained by optimization of the compaction and sintering process by tuning of the temperature and pressure, which will be the subject of future investigations.

Acknowledgments: Research supported by H2020 AMPHIBIAN (n. 720853) and Regione LombardiaINSTM, "Motorsport" projects.

\section{References and Notes}

1. D. Stoppels, J. Magn. Magn. Mater. 160, 323 (1996).

2. J. Y. Park and M. G. Allen, J. Micromechanics Microengineering 8, 307 (1998).

3. https://irapinnoresearch.wordpress.com/2015/03/26/report-summarysoft-magnetic-materials/.

4. J. M. D. Coey, Magnetism and Magnetic Materials, Cambridge University Press (2010).

5. G. Herzer, Acta Mater. 61, 718 (2013)

6. T. Ueno, H. Tsuruta, T. Saito, A. Watanabe, T. Ishimine, and K. Yamada, SEI Tech. Rev. 82, 9 (2016).

7. A. M. Leary, P. R. Ohodnicki, and M. E. McHenry, JOM 64, 772 (2012).

8. M. Drofenik, A. Žnidaršič, and I. Zajc, J. Appl. Phys. 82, 333 (1997)

9. H. Inaba, T. Abe, Y. Kitano, and J. Shimomura, J. Solid State Chem. 121, 117 (1996).

10. H. Yun, X. Liu, T. Paik, D. Palanisamy, J. Kim, W. D. Vogel, A. J. Viescas, J. Chen, G. C. Papaefthymiou, J. M. Kikkawa, M. G. Allen, and C. B. Murray, ACS Nano 8, 12323 (2014).

11. S. Lefebure, E. Dubois, V. Cabuil, S. Neveu, and R. Massart, J. Mater. Res. 13, 2975 (2011).

12. J. A. Lopez Perez, M. A. Lopez Quintela, J. Mira, J. Rivas, and S. W. Charles, J. Phys. Chem. B 101, 8045 (1997).

13. J. Wang, J. Sun, Q. Sun, and Q. Chen, Mater. Res. Bull. 38, 1113 (2003).

14. J. Zhang, L. Yu, S. Yuan, S. Zhang, and X. Zhao, J. Magn. Magn. Mater. 321, 3585 (2009).

15. C. Rath, K. Sahu, S. Anand, S. Date, N. Mishra, and R. Das, J. Magn. Magn. Mater. 202, 77 (1999).

16. A. L. Patterson, Phys. Rev. 56, 978 (1939).

17. E. Veena Gopalan, I. A. Al-Omari, K. A. Malini, P. A. Joy, D. Sakthi Kumar, Y. Yoshida, and M. R. Anantharaman, J. Magn. Magn. Mater. 321, 1092 (2009).

18. R. Arulmurugan, G. Vaidyanathan, S. Sendhilnathan, and B. Jeyadevan, J. Magn. Magn. Mater. 298, 83 (2006).

19. P. J. van der Zaag, J. J. M. Ruigrok, A. Noordermeer, M. H. W. M. van Delden, P. T. Por, M. T. Rekveldt, D. M. Donnet, and J. N. Chapman, J. Appl. Phys. 74, 4085 (1993).

20. T. Gaudisson, Z. Beji, F. Herbst, S. Nowak, S. Ammar, and R. Valenzuela, J. Magn. Magn. Mater. 387, 90 (2015).

21. B. D. Cullity and C. D. Graham, Introduction to Magnetic Materials, 2nd edn., Wiley-IEEE Press and John Wiley \& Sons (2009).

22. A. Skumiel, M. Kaczmarek-Klinowska, M. Timko, M. Molcan, and M. Rajnak, Int. J. Thermophys. 34, 655 (2013).

Received: 11 November 2017. Accepted: 26 January 2018. 